\title{
La presencia del profesor y su influencia para una educación significativa: hacia un enfoque mindfulness en educación*
}

\author{
Teachers' presence and their influence on significant learning: \\ Toward a mindfulness approach in education
}

\author{
Rodrigo Brito Pastrana ${ }^{a}$, Carolina Corthorn ${ }^{b}$ \\ ${ }^{a}$ Facultad de Ciencias, Universidad Mayor. \\ Correo electrónico: rodrigobritopastrana@gmail.com \\ ${ }^{\mathrm{b}}$ Facultad de Educación, Universidad Andrés Bello. \\ Correo electrónico: ccorthor@uc.cl.
}

\begin{abstract}
RESUMEN
Este artículo realiza una revisión teórica de las últimas investigaciones en relación a mindfulness en educación, especialmente relacionada con los aportes de esta práctica para el cuidado docente y para el cultivo de una presencia más plena por parte de los profesores y su influencia en la totalidad del proceso formativo, poniendo especial énfasis en el cultivo de algunas actitudes: atencionales (no juicio, mente de principiante y esfuerzo justo) y relacionales (amabilidad, empatía y compasión). A partir de la revisión de importantes investigaciones recientes, este trabajo propone un modelo cuyo propósito es orientar distintas intervenciones basadas en lo que en este trabajo llamamos un "enfoque mindfulness", enfatizando la necesidad de su carácter sistémico, más allá de eventos puntuales, con el propósito de influir en la totalidad del sistema educativo hacia una cultura del bienestar.

Palabras claves: consciencia, auto-cuidado, proceso formativo, actitudes atencionales, actitudes relacionales.
\end{abstract}

\begin{abstract}
This article presents a theoretical review of the recent research related to mindfulness in education, specifically that concerned with the contribution of this practice to the self-care of teachers and with how the cultivation of a fuller presence by teachers influences the formative process overall, putting special emphasis in the cultivation of certain attitudes: attentional (non-judgment, beginner's mind, and right effort) and relational (kindness, empathy, and compassion) attitudes. In this way, the study carries out a rigorous review of pertinent research and proposes a guiding model for diverse interventions based on what we call "mindfulness focus," emphasizing the need for a systemic view, beyond isolated events, with the purpose of influencing the education system overall toward a culture of well-being.
\end{abstract}

Keywords: consciousness, self-care, formative process, attentional attitudes, relational attitudes.

El presente artículo contó con financiamiento de la Universidad Mayor y del Fondo Interno Jorge Millas de la Universidad Andrés Bello. 


\section{PLANTEAMIENTO DEL PROBLEMA}

En la actualidad la educación en Chile se encuentra en un proceso de profunda reforma en todos sus niveles, aun cuando su dirección sea incierta. Las investigaciones realizadas en nuestro país son claras al demostrar que los profesores se enfrentan permanentemente a condiciones laborales estresantes y desgastantes, cuyas consecuencias son una progresiva desmotivación con su labor, una baja en su rendimiento, una merma de su creatividad, un alto ausentismo laboral, un clima interpersonal deteriorado, entre otras (Valdivia et al. 2003; Cornejo 2009). Un estudio sobre la salud laboral de los profesores en Chile (Valdivia et al. 2003) encontró que un $29,1 \%$ de los docentes presenta depresión mayor, y un 23,1\% trastornos ansiosos. Cornejo, por su parte, observó que los docentes presentaron altos niveles de agotamiento emocional $(26,6 \%)$, distancia emocional $(20,1 \%)$, falta de logro $(28,6 \%)$, estrés $(13,9 \%)$ y síntomas de depresión (13,2\%) (Cornejo 2009). Esto se refleja en el hecho de que nuestro país presenta una alta tasa de rotación docente con un abandono del $40 \%$ durante los primeros 5 años de ejercicio profesional (Gaete, Castro, Pino y Mansilla 2017).

Lo anterior es concordante con estudios a nivel internacional que señalan que entre $25 \%$ y $30 \%$ de los profesores afirman que su profesión es muy estresante o extremadamente estresante (Kyriacou 2001 y Unterbink et al. 2007; citados en Roeser, Skinner, Beers y Jennings 2012). El estrés laboral no sería un problema que afecta solo a la salud física y mental del docente. Las características propias del burnout (o desgaste profesional), tales como la irritabilidad, el desánimo y la hiperreactividad ante el estrés, son variables que representan graves interferencias en la relación profesor-alumno y, por ende, afectan la efectividad del proceso enseñanza-aprendizaje. Los docentes manifiestan la misma tendencia observada en otros profesionales con desgaste profesional, manifestando una gran distancia emocional como mecanismo de defensa para atenuar el impacto del estrés laboral (Masson 1990, citado en Arón y Milicic 1999). Estos aspectos tendrían efectos en el clima de aula y en los procesos e interacciones entre el educador y el estudiante, constituyendo uno de los factores relevantes para la calidad de la educación (Doherty, Forer, Lero, Goelman y LaGrange 2006). Sabemos que un profesor estresado y desgastado profesionalmente tendrá dificultad para atender en forma adecuada a las necesidades tanto emocionales como cognitivas de sus alumnos. A su vez, se traduce en bajos niveles de creatividad y flexibilidad en su desempeño, de manera que la implementación del currículum se vuelve más estrecha, rígida y estandarizada (Mañas, Franco, Gil y Gil 2014).

El problema educacional en nuestro país es reconocido, y ha motivado una serie de políticas, programas y proyectos de mejoramiento en los últimos 25 años, tales como el aumento de la cobertura, la reforma al estatuto docente, la incorporación de los objetivos transversales que debían ser perseguidos por todas las asignaturas y apuntaban principalmente a la formación ética y al desarrollo personal. En la última década, se ha avanzado en temas relevantes como, por ejemplo, la implementación de la carrera docente, la gratuidad parcial en la educación superior, la desmunicipalización de los colegios, entre otros (Arellano 2001). En la práctica, sin embargo, la educación parece haber sucumbido ante los requerimientos de las mediciones, lo que se ha traducido en un aumento de la exigencia curricular (aumento de horas de asignaturas como matemáticas y lenguaje) y en la incorporación de más mediciones estandarizadas introducidas desde otros contextos. El supuesto implícito parece ser que existe la creencia de que la educación se debe mejorar teniendo como foco casi exclusivo al alumnado, que es el que debiera aprender más 
contenidos, en más horas de clases, poniendo a prueba su conocimiento rindiendo más pruebas para ir evaluando este proceso.

Si bien es cierto que la importancia del rol del docente es reconocida, y que ha habido esfuerzos por mejorar los niveles de capacitación y aumentar estándares y criterios para evaluar el desempeño docente, sigue siendo desde una lógica productiva, es decir, de generación de mayor rendimiento académico/cognitivo, dejando de lado un aspecto central: el bienestar de la persona del docente. Paradójicamente, el hecho de que el foco esté puesto en la obtención de resultados de aprendizaje, y no en la calidad del proceso y de las interacciones, genera justamente menor rendimiento en los alumnos. La centralidad de la figura del profesor en el proceso de enseñanza-aprendizaje ${ }^{1}$ ha sido reconocida por muchos autores. De especial interés resulta el Modelo de Clase Pro-social propuesto por Jennings y Greenberg, que en síntesis sostiene que las competencias socio-emocionales y el bienestar del profesor es el motor principal que impulsa un flujo de influencias virtuosas, promoviendo una relación profesor-estudiante saludable, una mayor efectividad en el manejo de la clase y una implementación efectiva de un aprendizaje socio-emocional en los estudiantes. Estas tres variables, a su vez, repercuten en forma sinérgica generando un clima de clase beneficioso que sirve de plataforma para el cultivo, en el alumnado, de competencias socio-emocionales y académicas de utilidad para la vida (Jennings y Greenberg 2013). Para estas reflexiones, basta con enfatizar que el núcleo bisagra del sistema está puesto en la persona del profesor, en lo que podríamos denominar su modo de estar presente en el aula, presencia que de ser positiva es capaz de irradiar hacia su entorno y fomentar un tipo de formación y de relación basada en el bienestar y en la coconstrucción de una educación significativa.

En el contexto de una época como la nuestra, caracterizada por un afán por el rendimiento y por una aceleración creciente de los procesos (Han 2012), y de una educación como la actual, que en lo medular sigue estando dirigida por una lógica industrial orientada unilateralmente al cultivo de una inteligencia cognitiva, a la producción y a los logros académicos (Naranjo 2007; Robinson 2009), necesitamos pensar el proceso formativo desde lo que podríamos llamar una ética de la educación, en el sentido de la reflexión acerca de las destrezas concretas que podemos cultivar para generar una vida personal y comunitaria más feliz basada en la sinergia de la colaboración. Entenderemos la ética como una maestría más que un mero conocimiento, ya que esta última es más acorde a la habilidad de tocar un instrumento -por ejemplo- que al saber intelectual de normas, valores y orientaciones morales, que en sí mismas no garantizan aplicabilidad alguna (Varela 1998; Brito 2011; Brito 2015b).

Entre los múltiples abordajes que podemos encontrar para llevar a cabo el aterrizaje de esta ética de la educación, uno de los más prometedores ${ }^{2}$ es el que se basa en la implementación de prácticas de mindfulness en educación. Por esto, en lo que sigue, este trabajo se centrará en los aportes que en la actualidad se están realizando desde

A partir de ahora nos referiremos a este proceso como un "proceso formativo", ya que la expresión "enseñanza-aprendizaje" proviene de una tradición que enfatiza demasiado el aspecto cognitivo y técnico de la educación, mientras que el concepto de "formación" refiere a un proceso que implica la totalidad de las personas involucradas en él y releva la enorme importancia de las influencias implícitas en este proceso, ligadas a lo que veremos sobre el rol que cabe a la presencia de la figura del profesor(a) y su influencia en la emergencia de una educación verdaderamente significativa.

2 Gracias a una gran cantidad de investigaciones que respaldan este tipo de aproximaciones, en Reino Unido, por ejemplo, las prácticas de mindfulness son parte integral del sistema público de educación desde el año 2015 (ver más adelante). 
aproximaciones basadas en prácticas de mindfulness, específicamente en relación al autocuidado de los profesores y a los efectos benéficos que éste conlleva para el conjunto del proceso formativo. Con ese objetivo, primero se realizará una síntesis significativa de lo que en este ámbito se está investigando, para luego desarrollar una reflexión más amplia sobre las implicancias que puede tener la incorporación de un "enfoque mindfulness" en la totalidad del sistema y del proceso educativo. Sin embargo, antes de continuar conviene dejar establecida una definición de mindfulness que nos permita comprender qué implicancias tiene este tipo de práctica. Kabat-Zinn, fundador de esta propuesta, define mindfulness como "la consciencia no enjuiciadora que se despliega momento a momento, la cual se cultiva prestando atención de una manera específica: en el momento presente y de la manera menos reactiva y enjuiciadora posible, y con la máxima apertura del corazón" (Kabat-Zinn 2005).

\section{EVIDENCIA CIENTÍFICA SOBRE MINDFULNESS EN EDUCACIÓN}

Considerando la abundante evidencia científica que da cuenta de los efectos positivos del mindfulness en la reducción del estrés, y en el aumento del bienestar, la regulación emocional, la salud y las disposiciones prosociales (Baer 2003; Brown, Ryan y Creswell 2007; Greeson 2009; Grossman, Niemann, Schmidt y Walach 2004; Hoffman, Sawyer, Witt y Oh 2010), a nivel internacional se ha incrementado el interés en incorporar este tipo de estrategia para ayudar a los profesores a cultivar hábitos mentales que los ayuden a estar mejor preparados ante las demandas propias de su profesión. Asimismo, en su rol de formadores de niño(a)s y adolescentes, se plantea que están en una posición privilegiada para transmitir esta herramienta a sus alumnos, ya sea mediante el modelaje de una actitud y conducta que encarne consciencia y presencia plena (mindfulness) y/o a través de la instrucción directa a sus alumnos. Zenner, Herrnleben-Kurz y Walach, que realizaron un meta-análisis de 24 estudios para evaluar intervenciones basadas en mindfulness en el ámbito educacional, plantean que se trata de un área de investigación prometedora, con importantes implicancias tanto para el desarrollo cognitivo y social de los niños como para la salud mental del personal educativo. A su vez, estos autores plantean que un buen modelo de intervención se enfocaría, en primer lugar, en la incorporación de la práctica de mindfulness por parte de los docentes, para que luego ellos puedan promoverla en sus alumnos, de las maneras ya descritas, con estrategias apropiadas para la etapa del desarrollo en que se encuentran los estudiantes (Zenner, Herrnleben-Kurz y Walach 2014).

Los estudios que dan cuenta de los beneficios del mindfulness para los docentes van en aumento. Por ejemplo, Weare señala, en base a una revisión de 13 estudios, que a través de intervenciones basadas en mindfulness se reduce el estrés docente, mejora la salud mental, aumenta el bienestar, la bondad y la compasión, mejora la salud física, aumenta el rendimiento cognitivo y el rendimiento laboral (Weare 2014). Por su parte, Delgado et al. encontraron efectos del mindfulness en la autorregulación emocional y prevención del estrés, y mejora en la calidad de vida (Delgado et al. 2010). En otro estudio enfocado en docentes, Franco, Mañas, Cangas, Moreno y Gallego encontraron que los niveles de angustia psicológica se vieron reducidos significativamente (Franco, Mañas, Cangas, Moreno y Gallego 2010), y Mañas, Franco y Justo observaron que, después de un programa de entrenamiento de mindfulness, los niveles de estrés docente y los días de baja laboral por 
enfermedad revelaban una reducción significativa (Mañas, Franco y Justo 2011). Franco et al. mostraron una reducción en el malestar docente y Kemeny et al. encontraron reducción de emociones negativas y de sentimientos de depresión, y aumento en los estados de ánimo positivos (Franco et al. 2010; Kemeny et al. 2011).

Sintetizando la evidencia expuesta habría, según Shapiro, Brown y Astin, tres grandes áreas de impacto (Shapiro, Brown y Astin 2011). En este sentido, la introducción de prácticas de mindfulness para los profesores tendría los siguientes efectos positivos: 1) Mejora las habilidades cognitivas, en términos de una mejora en la capacidad de concentración, atención y creatividad; 2) mejora la autorregulación emocional y reduce/ previene síntomas como el estrés, la ansiedad y la depresión; y 3) fomenta un desarrollo humano más integral, mejorando las relaciones interpersonales y la capacidad para la autocompasión y la compasión hacia los demás.

Por su parte, Meiklejohn et al. han llevado a cabo una revisión de distintos programas de autocuidado docente, destacando entre otros: el Mindfulness-Based Wellness Education (MBWE), en Toronto (Canadá); el Cultivating Awareness and Resilience in Education (CARE), en Denver (USA); y el Stress Management and Relaxation Techniques (SMART), en Colorado y Vancouver (USA) (Meiklejohn et al. 2012). Todos estos programas, y otros no mencionados aquí, demuestran tener un resultado común: que los profesores que practican sistemáticamente mindfulness desarrollan cualidades positivas en su actitud, presencia e interrelación con los estudiantes y sus colegas, contribuyendo a generar una atmósfera educativa óptima para el proceso formativo. Es importante mencionar, además, que en todos estos programas queda en evidencia que la efectividad de las prácticas de mindfulness aumenta significativamente cuando no son consideradas como una mera técnica, sino como un estilo de vida que se irradia en la sala de clases, pero también fuera de ella, en todos los ámbitos de la vida personal e interpersonal de los profesores.

Según otras investigaciones, las prácticas de mindfulness favorecen en el docente un mejor manejo de aula y mejor autoestima. A su vez, algunos profesores señalan que la atención plena les ayudaría a tener una visión más holística del currículo y conceptos clave, disminuyendo la sensación de agobio por el gran número de contenidos que deben abordar (Albrecht, Albrecht y Cohen citado en Cebolla, García-Campayo y Demarzo 2014). Esto significa que las prácticas de mindfulness no sólo repercuten en el bienestar personal e interpersonal en contextos educativos, sino que ayuda a los profesores a encarnar de un modo más lúcido y discriminativo el currículo y las múltiples exigencias pedagógicas a las que suelen estar expuestos. Esta capacidad para filtrar y adecuar contenidos y métodos es clave a la hora de pensar en una educación que los estudiantes la sientan como significativa ${ }^{3}$.

Por otra parte, Flook, Goldberg, Pinger, Bonus y Davidson realizaron un estudio experimental piloto en el cual se implementó una versión adaptada a profesores del programa (MBSR- Programa de Reducción de Estrés basado en mindfulness, por sus siglas en inglés). Los resultados dieron cuenta de una mejora en síntomas de salud mental y reducción

Otra distinción interesante es la que podemos hacer entre una educación de "calidad" y una "significativa". El concepto de "calidad" ha sufrido un uso tan indiscriminado que ha pasado a enredar más que aclarar las metas de la educación, principalmente por su alto grado de ambigüedad y por su enraizamiento en una tradición demasiado ideologizada y desligada de los contextos de la vida real. En cambio, el concepto de "significativa" tiene el potencial de devolvernos a un cierto sentido común. Tomando distancia de la definición de Ausubel, según la cual un aprendizaje es significativo cuando lo que el alumno aprende coincide con conocimientos previos (Ausubel 1983), entenderemos en este contexto que una formación es significativa cuando es pertinente: a los problemas del mundo real en que vivimos, a los contextos de los profesores y del alumnado, a las posibilidades de los actores educativos, etc. 
de burnout -o desgaste profesional-, mejora en la calidad de la interacción profesoralumnos observada en aula y aumento en los niveles de auto-compasión. En contraste, los participantes del grupo control presentaron niveles marginalmente significativos de aumento en niveles de burnout (Flook, Goldberg, Pinger, Bonus y Davidson 2013).

En 2015, en una forma que podríamos considerar paradigmática, se publica en Reino Unido el informe Mindful Nation UK del Parlamento inglés. Este informe muestra evidencia contundente acerca de los beneficiosos efectos que tienen las prácticas de mindfulness para el conjunto de la comunidad educativa, razón por la cual se propone incorporarlas en el sistema público de educación, así como en los sistemas públicos de salud y justicia. Este paso se fundamenta en un gran número de investigaciones realizadas en diversas universidades británicas, cuyos resultados coinciden en algunos puntos centrales: las prácticas de mindfulness por parte de los profesores contribuyen a reducir sus niveles de estrés, a aumentar su nivel general de bienestar y a cultivar una forma más auto-compasiva de tratarse a sí mismos (Beshai, McAlpine, Weare y Kuyken 2016). En lo fundamental, este reporte ratifica lo que señalan la mayoría de las investigaciones presentadas, incluyendo los beneficios que las prácticas de mindfulness tendrían para los estudiantes, principalmente en relación a la auto-regulación ejecutiva y emocional y a la prevención de sintomatología en los espectros del estrés, la ansiedad y la depresión (Mindful Nation UK 2015).

En relación a los profesores, este reporte, plantea que la implementación de este tipo de programas en las escuelas depende considerablemente de la calidad y experiencia de la práctica de mindfulness de los docentes, lo cual puede llevar varios años de compromiso personal (Mindful Nation UK 2015). Esta conclusión del reporte es, en el contexto del presente trabajo, muy relevante, pues orienta una comprensión de las prácticas de mindfulness más en la línea del cultivo de una forma de vida que en el sentido de la aplicación de una mera técnica para la resolución de problemas puntuales. Esto es, precisamente, lo que hace que autores relevantes como Kabat-Zinn se atrevan a hablar de un "cambio de paradigma" a partir del enfoque mindfulness, el que consistiría en un cambio de visión o de actitud en/ante el mundo hacia una comprensión de los fenómenos en términos de totalidades y de interconexiones:

Cuando nuestra mente cambia, aparecen nuevas posibilidades. Todo cambia, de hecho, cuando contemplamos las cosas simultáneamente desde niveles diferentes, como cuando vemos, por ejemplo, su plenitud y su conexión o su individualidad y su separación. En tal caso, la amplitud de nuestro pensamiento se expande (Kabat-Zinn 2016).

Para sintetizar lo dicho hasta aquí, según Arguís (En Cebolla, García-Campayo y Demarzo 2014)

En general, los resultados de todas las investigaciones son satisfactorios, y reflejan que la práctica de la atención plena produce efectos positivos sobre las variables estudiadas. No obstante, hay que señalar que muchos de estos estudios han sido realizados con muestras pequeñas de población, por lo que hay que tener una gran cautela en la generalización de los resultados. La conclusión más evidente es que se requieren muchos más estudios, con muestras más amplias de población y con diseños de investigación muy refinados que, en un futuro, nos permitan disponer de más evidencias firmes en este terreno (64). 
Así, vemos que, si bien contamos con bastante evidencia acerca de los beneficios del mindfulness en contextos educacionales, ésta es aún preliminar. En nuestra opinión, esto implica dos desafíos; en primer lugar, que necesitamos investigar con mayor profundidad cómo las prácticas de mindfulness pueden, a través de la presencia/actitud del profesor, influir en la totalidad del sistema educativo; en segundo lugar, que necesitamos contar con una fundamentación teórica (susceptible de implementación) suficientemente rigurosa y que trascienda la especificidad de los beneficios que la aplicación de una técnica puede conllevar, orientada hacia una reflexión acerca del sentido de la educación y del educar en general en el contexto de nuestra época actual. Esto requiere un salto reflexivo, en el que podamos complementar la investigación de intervenciones puntuales basadas en prácticas de mindfulness, con un desarrollo teórico-práctico sistémico en el que podamos re-pensar el proceso educativo completo desde un enfoque mindfulness considerando, por supuesto, las peculiaridades de los diferentes contextos socio-culturales.

\section{HACIA UN MODELO TEÓRICO ORIENTADOR DEL ENFOQUE MINDFULNESS EN EL CONTEXTO EDUCATIVO}

A partir de la revisión de la evidencia proponemos un modelo teórico de la práctica mindfulness en el contexto educativo, considerando las principales variables que se verían impactadas a partir de la práctica, tanto de los docentes y estudiantes, como también a nivel de las interacciones y del clima de aula.

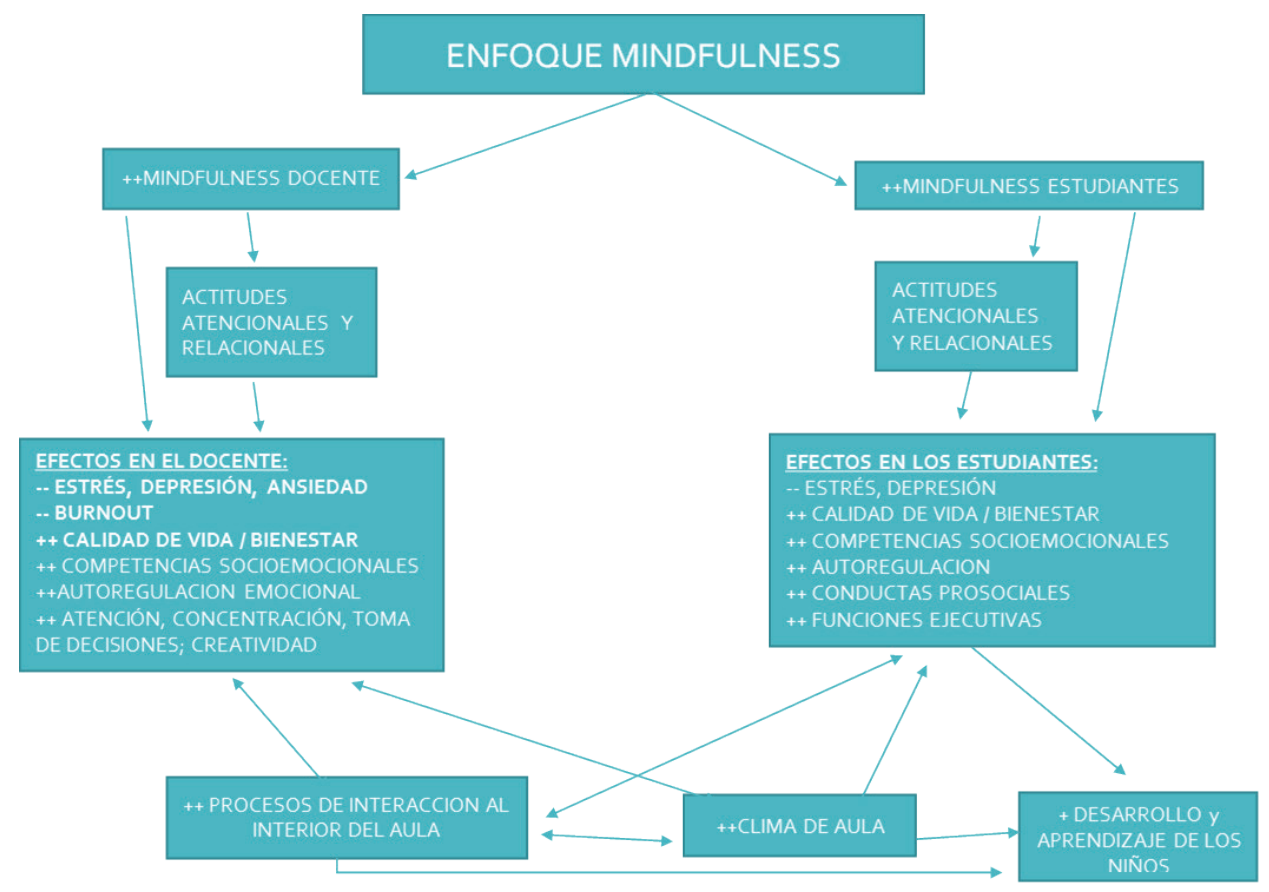


En esta figura damos cuenta de los componentes del modelo, remarcando que un enfoque mindfulness en el contexto educativo tendría impacto tanto en los docentes como en los estudiantes, aumentando niveles de mindfulness, específicamente en actitudes atencionales y relacionales que se cultivan mediante esta práctica. La evidencia da cuenta de que el mindfulness tendría efectos en las variables de los docentes que planteamos en el modelo, y suponemos que esto a su vez impactaría en los procesos de interacción al interior del aula y en el clima de aula. Todo esto tendría efecto en los estudiantes. Por otro lado, el aumento de mindfulness y actitudes relacionadas en los mismos estudiantes, tendría también efectos en variables de los alumnos, y en los docentes, mediante su influencia en el clima y la interacción.

Desde nuestra perspectiva, un enfoque mindfulness implica la tarea de pensar y de concebir una forma concreta de cultivar la actitud o presencia de los profesores, entendiendo por actitud el conjunto de disposiciones implícitas de un individuo en todas las dimensiones de su existencia (modos de pensar, de sentir, de relacionarse, de actuar, etc.) que se expresan en un modo específico y habitual de estar presente en el mundo (Brito 2015a). En esta misma línea estarían autores como Robinson, al mostrar con ejemplos, a través de personas concretas, cómo la capacidad de un profesor de estar atento a sus estudiantes -junto con su creatividad-, puede ser el hito decisivo para que un estudiante descubra y se dedique a aquello que lo apasiona y, al mismo tiempo, para lo cual tiene talento; esta capacidad ha sido definida por Robinson como "estar en su elemento" (Robinson 2009). Naranjo, por su parte, señala que sólo los profesores que cultiven su propia presencia consciente pueden formar estudiantes con más consciencia (Naranjo 2007). Como señala Leonard (citado en Naranjo 2007)

La pregunta que más a menudo nos hacemos es qué: ¿qué materias hemos de enseñar? Cuando la conversación se hace más profunda, pasamos a preguntarnos por el cómo: ¿qué métodos y técnicas son necesarias para una buena enseñanza? Ocasionalmente, cuando se hace aún más profunda, llegamos a preguntar el por qué: ¿con qué propósito y para qué enseñamos? Pero rara vez nos preguntamos por el quién: ¿cómo puede la calidad de mi ser [cursivas de los autores] determinar la manera en que me relaciono con mis estudiantes, mi tema, mis colegas, mi mundo? (221).

Una dimensión clave para comprender y cultivar una actitud adecuada en los profesores es el autocuidado. Esto es muy relevante, pues significa que no tenemos que esperar a que las políticas públicas en educación ${ }^{4}$ tomen consciencia de su significación, pudiendo intervenir desde ya concientizando y entregando un repertorio de prácticas acerca del sentido de una cultura del bienestar en educación y de cómo los profesores pueden cuidarse y cultivar una actitud adecuada para fomentar una atmósfera y un tipo de relación idónea para un proceso formativo significativo, creativo y estimulante para todos.

\footnotetext{
Conviene recordar que los sistemas educativos de todo el mundo tienen un rasgo común de difícil abordaje: suelen tener lógicas y prácticas institucionalizadas muy rígidas y de compleja modificación. De hecho, son muchos los autores que sostienen que mientras la sociedad y la economía han cambiado vertiginosamente en los últimos 100 años, la educación actual en la mayor parte del planeta sigue pensándose y haciendo básicamente lo mismo: educar para un mundo meramente industrial. Esto puede resultar muy desalentador para quienes tenemos un fuerte interés en generar transformaciones significativas en este contexto, sin embargo, las mismas prácticas de mindfulness pueden ayudarnos a sostener un esfuerzo adecuado, con paciencia y con una clara consciencia de que sólo podemos aportar un grano de arena desde el lugar en que nos toca participar.
} 
Esta actitud "adecuada" queda definida en forma precisa por Kabat-Zinn al comprender mindfulness como el cultivo de la capacidad para prestar atención en forma intencional a la experiencia del momento presente sin juzgar y con aceptación (Kabat-Zinn 2016). Hay en esta definición dos aspectos fundamentales: el primero tiene que ver con que mindfulness entrena nuestra atención para estar más enfocada en el momento presente durante más tiempo; el segundo, que emerge sobre la base del primero, se refiere a que mindfulness nos permite cultivar una actitud de amabilidad y compasión hacia nosotros mismos y hacia los demás (Germer \& Siegel 2012).

En Chile, la investigación sobre los efectos de las prácticas de mindfulness en el quehacer docente es muy incipiente, por lo que no encontramos aún reflexiones y aplicaciones que estén dentro de esta ética que aquí se intenta bosquejar. Esto hace que las reflexiones que siguen no pasen de ser exploratorias. A continuación, abordaremos algunos "principios orientadores" que, a nuestro juicio, deberían ser considerados a la hora de reformular el proceso formativo desde un enfoque mindfulness.

Lo primero es que una reformulación de esta naturaleza supone partir de abajo hacia arriba, desde la perspectiva de su incorporación concreta al quehacer educativo cotidiano, no respecto de la vía de acceso de esta perspectiva en la institución educativa, acceso que debiera comenzar por una sensibilización y un proceso de asimilación por parte de las autoridades educativas (de arriba hacia abajo). Esto implica implementar prácticas y formas de relación en las diferentes dimensiones educativas (autocuidado docente, autocuidado estudiantil, interrelaciones entre los diferentes actores educativos, equilibrio de tiempos entre docencia y tiempos libres de rendimiento, etc.) de acuerdo a lo que ya sabemos acerca de mindfulness. Lo principal que sabemos es que las prácticas de mindfulness ayudan a cultivar una actitud más basada en el ser que en el hacer, así como un modo de relacionarse con uno mismo y con el entorno más amable y compasivo. En este sentido, tendríamos que atrevernos a ver qué es lo que sucede si le damos más espacio al poder (trans)formativo de la presencia de los profesores desde un entrenamiento en estas prácticas.

Luego, consideramos importante complementar tiempos que actualmente se destinan a consejos y reuniones focalizados exclusivamente en lo académico-administrativo con tiempos para reunirse como equipos de colaboración para practicar y abrir espacios para observar y experienciar qué es lo que se está haciendo y qué sentido tiene, para entrar en contacto con la significatividad o no significatividad del quehacer formativo. Esto implica mantener vivas ciertas preguntas fundamentales, tales como: ¿para qué estamos haciendo y/o enseñando tal o cual contenido? ¿Es ésta la mejor manera que tenemos de hacerlo? ¿Qué necesitamos como profesores para mantenernos claros y motivados con nuestra labor? ¿Qué están necesitando nuestros estudiantes para sentirse mejor con ellos mismos y para poder interactuar de mejor manera con el mundo que les toca vivir? ¿Cuáles son los verdaderos problemas a los que se tendrán que enfrentar nuestros estudiantes? Creemos que si se instauraran este tipo de encuentros no tardaríamos mucho tiempo en ver cómo irían emergiendo algunos frutos significativos, revitalizando todo el proceso formativo y re-orientándolo en direcciones más pertinentes a la realidad local.

Por otra parte, es imprescindible comenzar a flexibilizar los límites de los sistemas educativos, los que suelen estar demasiado ensimismados y excesivamente pendientes de las mediciones y de los rankings, perdiendo la oportunidad de mirar "hacia afuera" para ver qué está pasando en el mundo real, cuáles son nuestros mayores problemas como sociedad y como cultura. ¿Es ajeno, acaso, al proceso formativo el alto nivel de depresión y de 
adicciones que encontramos en nuestras sociedades "desarrolladas"? ¿No tiene nada que ver con este proceso el hecho de que estemos padeciendo a nivel ecológico esta patología planetaria llamada “calentamiento global”? ¿La educación no tiene ninguna responsabilidad en la construcción de esta desquiciada sociedad del consumismo? Esto implica que el sistema educativo adopte, en su conjunto, una actitud de apertura, atención y compasión por el contexto en el que está inserto; así como el no olvido de que toda formación significativa debe estar orientada a vivir más plenamente en el mundo y no, solamente, a tener mayor éxito material; o dicho en forma concisa: toda formación significativa es transformativa.

Además, necesitamos incorporar en la educación la convicción de que no se trata solo de enseñar y aprender, sino de generar una actitud tanto hacia uno mismo como hacia el mundo y que este proceso es complejo precisamente porque requiere "comenzar por casa"; es decir, por el cultivo de una actitud propicia en los propios profesores y equipos directivos, incluso antes de intentar formarla en los estudiantes. Es precisamente en este punto donde podemos visualizar mejor en qué consiste eso que antes llamamos "cambio de paradigma": llevar a cabo un proceso (trans)formativo desde un enfoque mindfulness. Desde este enfoque, hay ciertas actitudes que tienen una gran relevancia para la vida, independientemente del futuro quehacer profesional y de los eventuales logros materiales. En este sentido podemos hablar de dos ámbitos actitudinales primordiales, uno que podríamos situar del lado de la atención, y otro que podríamos situar en el plano de las relaciones.

\subsection{ACTITUDES ATENCIONALES}

Este tipo de actitudes se refieren a nuestro modo concreto de estar presentes desde nuestra unidad mente-cuerpo, momento a momento en diferentes contextos. En palabras de Siegel: "Vivir plenamente en el presente es una forma de arte que libera la mente, alivia el sufrimiento interior y desarrolla la presencia en nuestra vida. Esta presencia es fundamental para ayudar a los demás a alcanzar la salud" (Siegel 2012).

Se sabe que nuestro modo de estar presente es algo cambiante y modificable por medio de entrenamientos específicos, como las prácticas de mindfulness: "cuando se practica la meditación durante varios años, esos cambios positivos cambian la estructura física del cerebro" (Williams y Penman 2013). En la actualidad existe evidencia que demuestra cómo estas prácticas no sólo producen cambios funcionales en el cerebro, sino también estructurales, lo que se expresa en cambios concretos en nuestro modo de estar presentes; por ejemplo, reduciendo la tendencia natural de nuestra mente a distraerse y viajar en el tiempo, debido principalmente a la neuroplasticidad cerebral (Bachrach 2014). A continuación, nos limitamos a señalar algunas actitudes atencionales (entre otras ${ }^{5}$ ), a nuestro juicio, claves en el contexto educativo que se cultivan desde un enfoque mindfulness, todas ellas tratadas en diversos libros por Kabat-Zinn (Kabat-Zinn 2013, 2016), así como por otros autores (Brito, Segú, y Araya 2014).

\section{a. Actitud de no juicio}

Habitualmente nuestra mente está generando un flujo constante de pensamientos, la mayoría de los cuales puede ser entendido como juicios evaluativos acerca de la experiencia

Kabat-Zinn, plantea que hay siete actitudes atencionales básicas que se cultivan con la práctica de mindfulness. Las otras cuatro son: aceptación, soltar, paciencia y confianza (Kabat-Zinn 2016). 
presente. Ejemplos concretos pueden ser: "esto me encanta, debería suceder más seguido", "esto me molesta mucho, ojalá se termine ya”, "iqué persona más desagradable!", "seguro que ahora va a decirme lo mismo de siempre", etc. Al hacer esto nuestra mente crea un mundo narrativo en donde nuestras posibilidades quedan pre-definidas, convirtiéndose literalmente en profecías autocumplidas, limitando nuestro repertorio de acción en una situación determinada. Las prácticas de mindfulness nos permiten ir reconociendo poco a poco estos juicios habituales, dándonos la oportunidad de observarlos y, tal vez, disolverlos antes de que se transformen en narrativas rígidas y dominantes, aportándonos mayor libertad y un mayor repertorio de respuestas en sintonía con la situación en la que estamos. Si miramos con atención los conflictos habituales que surgen en el contexto educativo, la mayoría de ellos tiene que ver con pre-juicios que profesores y estudiantes tienen respecto a sí mismos y hacia los demás, lo que produce una cadena de malos entendidos y una merma en la calidad de la comunicación y de las relaciones interpersonales. Si directivos, profesores y estudiantes pudieran estar presentes de un modo menos (pre)juicioso, sin duda que podríamos reducir una buena parte de los problemas que hoy aquejan a nuestras instituciones educativas, tales como la violencia y la desconfianza que suelen predominar.

\section{b. Actitud de mente de principiante}

Esta es una actitud muy importante y útil, que la podemos observar sin excepción en los niños pequeños. Si los observamos detenidamente podemos reconocer que ellos están motivados por el asombro y la curiosidad, no aferrándose a prejuicios ni ideologías de ningún tipo. Los adultos, en cambio, tendemos a actuar mucho más desde nuestras expectativas (sean éstas positivas o negativas) repitiendo una y otra vez cursos de acción y/o formas de pensar que suelen mantener los problemas en lugar de resolverlos. Las instituciones educativas, dado su alto nivel de rigidez y de autoexigencia suelen pasarse años dándole vuelta a las mismas cosas sin producir cambios significativos. Las prácticas de mindfulness, con el tiempo, van generando una mayor apertura y un mayor nivel de creatividad y de osadía, lo que permite generar nuevas ideas y nuevas acciones en relación con problemas antiguos. Es evidente que si la comunidad educativa cultiva una mente de principiante es probable que encuentre estrategias más hábiles y beneficiosas para resolver casi cualquier desafío que se presente, como por ejemplo tomar mejores decisiones respecto del currículum, de los métodos de enseñanza o de los modos de resolver conflictos cotidianos. De este modo, estaríamos menos expuestos a probar a ciegas una metodología tras otra, o mantener la porfiada esclavitud respecto de las mediciones oficiales que suelen direccionar reductivamente todo el proceso formativo, con todas las consecuencias que esto conlleva para todos los actores implicados.

\section{c. Actitud de esfuerzo justo}

En nuestra cultura del rendimiento esta actitud puede resultar muy controversial y se puede prestar para malos entendidos. Creemos que para comprenderla en su justa dimensión es conveniente describir cómo funciona en general la cultura laboral educativa, al menos en Chile. En pocas palabras, diríamos que se caracteriza por un alto nivel de autoexigencia y de desgaste, que no necesariamente se traduce en resultados exitosos, en todos los sentidos que esta palabra puede tener. Por supuesto que aquí hay responsabilidades compartidas: 
por una parte, tenemos un sistema muy demandante que impone a los profesores tener que llevar trabajo para la casa y reducir el tiempo de descanso y de encuentro dentro de sus jornadas. Por otra, tenemos a profesores que no tienen mucha conciencia de la importancia de hacer respetar sus límites y que no saben en concreto cómo cuidarse de mejor forma. A modo de ejemplo, no por trabajar muchas horas seguidas vamos a cumplir mejor con nuestros objetivos pedagógicos. También en este punto las prácticas de mindfulness nos pueden ayudar, a través de un entrenamiento adecuado, a regular mejor nuestro esfuerzo para aprender el difícil arte de saber cuándo y cómo tenemos que esforzarnos, y cuándo y cómo sería mejor soltar y dar espacio para no actuar y para ponerse en una actitud más abierta y receptiva. Como dice el Tao Te King, el sabio sabe perfectamente que la mejor forma de hacer es, simplemente, ser (permanecer presentes) (Tse 1990).

\subsection{ACTITUDES RELACIONALES}

Aquí nos encontramos con un tipo de actitudes de especial importancia para la labor docente, dado que se trata de un quehacer en donde el tipo de relación que se establezca con los estudiantes, y entre colegas, es definitorio de la atmósfera en la que se desplegará el proceso formativo, repercutiendo directamente en la construcción de una formación más o menos significativa. Como señala Marchesi (Citado en Canales y Rovir 2016) "Un docente capaz de sentir empatía tiene la sensibilidad necesaria para ser consciente del estado personal del alumnado, de tal manera que los matices de su expresión no le pasarán desapercibidos". Es importante mencionar que este tipo de actitudes sólo pueden darse sobre la base del cultivo de una atención al momento presente que tenga consistencia y estabilidad en el tiempo, pues la atención plena es la tierra firme sobre la cual pueden emerger todas las otras actitudes, incluidas las que revisaremos a continuación.

\section{a. Actitud amable}

Se trata de una actitud en donde la disposición hacia los demás (y hacia uno mismo) se caracteriza por un tono cálido, cuidadoso y aceptador, independientemente de si estamos o no de acuerdo con el modo de pensar o de actuar de la otra persona. En el fondo, con esta actitud deseamos sinceramente contribuir al bienestar de quienes nos rodean y de nosotros mismos. Esto se traduce, en concreto, en un modo de escucha atenta y en una forma de hablar generadora de confianza, no violenta o enjuiciadora. En la educación actual vemos los esfuerzos que se hacen por generar este tipo de relaciones; sin embargo, se hace desde una lógica intelectualista basada en la enseñanza de valores. El problema de esta lógica es que un valor, por definición, no es más que una explicación o, en el mejor de los casos, una declaración de buenas intenciones que no necesariamente se traduce en una acción y en una disposición como la que estamos mostrando. Una actitud amable tiene que ser entrenada sistemáticamente y debe estar en continua revisión por la comunidad educativa en su conjunto. La potencia de esta actitud (y en general de todas) es que tiende a irradiarse y a contagiar a los demás, por lo que partiendo de un círculo pequeño de personas que se comprometan a cultivarla puede tener un efecto beneficioso para todo el sistema en el mediano y largo plazo. En palabras de Stalhl y Goldstein, amabilidad es la "cualidad en la que uno desea realmente el bien del otro, es decir, que esté sano, libre de daño y de miedo" (Stalhl y Goldstein 2010). 


\section{b. Actitud empática}

Uno de los primeros psicólogos que hizo de esta actitud el centro de las características de un buen terapeuta fue Carl Rogers (Rogers 1989). Significa la capacidad para sentir y comprender la experiencia del otro desde su mundo particular de referencia, en lugar de tratar a los demás desde nuestra propia medida de cómo las cosas deberían ser. En el fondo, esta actitud nos permite descentrarnos de nosotros mismos y dar cabida a la experiencia del otro, posibilitando una acción más efectiva, por ejemplo, en la resolución de un conflicto. $\mathrm{Si}$ bien estas ideas surgieron inicialmente para orientar la formación de terapeutas, hoy sabemos que hay mucho en común entre la labor de éstos y la de los profesores, en la medida en que nos relacionamos con otros seres humanos buscando su crecimiento y desarrollo. Siegel se refiere a esta actitud con la palabra "resonancia", la que según el autor "exige vulnerabilidad y humildad. No sabemos dónde nos llevará una interacción y no podemos controlar su resultado. La resonancia nos sumerge así en lo desconocido y nos sitúa cara a cara con la incertidumbre" (Siegel 2012). Nuestro sistema educativo está muy necesitado del cultivo de este tipo de actitudes en todos los actores educacionales. Es muy probable que toda la atmósfera emocional y relacional mejoraría significativamente si desarrollamos cada vez más la capacidad para comprender cómo sienten y significan el mundo los demás. Entonces, tal profesor deja de ser percibido como "antipático" para aparecer en su inseguridad y timidez, o tal compañero deja de ser considerado "agresivo" para ser visto como alguien que tal vez recibe poco afecto de su familia o se siente muy invisibilizado e inseguro, teniendo la necesidad de llamar la atención.

\section{c. Actitud compasiva}

Esta actitud ha recibido especial atención de la investigación en mindfulness en los últimos años, evidenciando su relevancia y utilidad relacional a la hora de generar vínculos de mayor calidad humana. Según Araya y Moncada, siguiendo a Germer y Siegel (Araya y Moncada 2016; Germer y Siegel 2012)

[...] la compasión implica un sentimiento de bondad, cuidado y comprensión por las personas que sienten dolor, junto con el deseo emergente de aminorar su sufrimiento, implicando un reconocimiento de compartir la condición humana, frágil e imperfecta (Germer y Siegel 2012).

Según lo anterior, hay en esta actitud al menos dos elementos propios que la distinguen de otras actitudes (incluidas las dos anteriores). Primero, que la compasión surge cuando nos damos cuenta de que hay sufrimiento en nosotros mismos o en otra persona. Segundo, la compasión conlleva una tendencia a hacer algo para aliviar ese sufrimiento, yendo más allá de un mero "ponerse en el lugar de otro" . Además, implica resaltar nuestra condición de vulnerabilidad e imperfección, a contracorriente de la tendencia imperante en las culturas educativas de nuestro país a sobrevalorar la autoexigencia y el perfeccionismo

Esta es una de las diferencias centrales entre la compasión y la empatía: la tendencia que conlleva la compasión a realizar una acción concreta para aliviar el sufrimiento propio y/o de otro, manteniendo siempre una vigilancia sobre los límites propios y el autocuidado. De ahí que la investigación actual ponga en estrecha relación esta actitud compasiva con la acción altruista (Ricard 2016). 
como virtudes, con todas las implicancias que esto tiene respecto de la intolerancia que hay ante el error y del fracaso, experiencias que, por lo demás, tienen un gran potencial para el aprendizaje y el desarrollo personal y colectivo.

Según Neff, investigadora estadounidense experta en este tema, la autocompasión tiene tres componentes básicos interconectados:

a) Implica una actitud de amabilidad y comprensión cuidadosa (tal como lo describimos anteriormente).

b) Implica una toma de consciencia de nuestra humanidad compartida, es decir, una comprensión de corazón de pertenecer a una misma familia humana, más allá de cualquier diferencia.

c) Implica cultivar una atención plena capaz de ver y comprender el sufrimiento de un modo profundo y sistémico, más allá de una acción o conducta específica aparentemente incomprensible. Nuestro sistema educativo en su totalidad necesita especialmente del cultivo de esta actitud, ya que hay demasiado sufrimiento en todos los actores implicados, desde el desgaste profesional de los profesores hasta el sentido de inadaptación y la desmotivación de nuestro alumnado. (Neff 2012).

\section{CONCLUSIÓN Y DISCUSIÓN}

No exageramos al afirmar que uno de los principales problemas de nuestra educación actual consiste en su des-humanización, es decir, en la ausencia y olvido de construir relaciones significativas basadas en una mayor atención y en una actitud más amable y comprensiva. Tampoco lo hacemos si decimos que una de las puertas de entrada clave para aportar a corregir este desvío consiste precisamente en comenzar por introducir a nivel sistémico una mirada y un entrenamiento desde lo que podríamos llamar un enfoque mindfulness en educación, según lo ya planteado. Este es, en el fondo, el diagnóstico que propone Naranjo al señalar que "la educación patriarcal, que es la que conocemos desde siempre, es una educación predominantemente intelectual en la que los demás aspectos del ser humano son desestimados" (Naranjo 2007).

Luego de décadas intentando mejorar el currículum y dando énfasis a las transversalidades en educación, instalando y desinstalando miradas y modos de hacer las cosas, tenemos que reconocer que, al menos en Chile, no ha ocurrido un cambio fundamental y no hemos podido construir una educación significativa que considere el continuo cambio y la creciente imprevisibilidad de los escenarios futuros a los que se enfrentarán nuestros estudiantes. Precisamente es éste uno de los cambios más importantes a nivel global que reclama un giro paradigmático en educación: hemos pasado de un mundo en el que el futuro era medianamente predecible bajo una lógica industrial en la que cada cual es educado para calzar en alguna pieza del gran engranaje, a uno marcado por una incalculable complejidad que requiere desarrollar habilidades y actitudes basadas en una gran comprensión de diferentes contextos, en una adecuada capacidad para la autoregulación emocional (Cullen y Brito 2016) y en el desarrollo de un alto nivel de creatividad (Robinson 2009). En esta dirección se encuentra también la necesidad de hacer un cambio 
de énfasis, desde una educación basada en el aprendizaje de conocimientos y destrezas, hacia una que sea fuertemente complementada con una (trans)formación de actitudes para el mundo complejo que nos toca vivir, las que van en las líneas antes señaladas. Este tipo de formación requiere transitar a un nuevo paradigma en educación, que podemos llamar, siguiendo a Casassus, paradigma constructivista (Casassus 2002). En este nuevo escenario, las personas y el tipo de relaciones que establecen es la clave de todo el proceso de enseñanza-aprendizaje. Por eso es que un foco en la actitud, en el autocuidado y en el bienestar de los profesores se vuelve crucial en este nuevo escenario:

Así como la vida procede sólo de la vida, la conciencia sólo puede ser despertada por la conciencia. Se necesita, por lo tanto, de un tercer elemento entre las ramas del currículum clásico y de esa educación en los valores que se pretende llevar a cabo a través de las transversalidades: la transformación del educador (Naranjo 2007).

En estas reflexiones hemos distinguido en forma implícita las palabras "educación” y "(trans)formación". Es importante aclarar este punto, aunque no sea más que para abrir un espacio para hacernos preguntas necesarias sobre la educación que necesitamos y sobre el rol que a los profesores les compete en ella. La noción de formación, a diferencia de la de educación, pone un énfasis en la transformación de la persona total del estudiante en el contexto de una relación significativa que genere las condiciones para que esto sea posible. Por tanto, va mucho más allá de la mera adquisición de conocimientos y de habilidades. Nos parece especialmente sugerente el pensamiento de Gadamer (Gadamer 1994) a este respecto, que desde la tradición humanista occidental retoma para las disciplinas humanistas contemporáneas su misión original de transformar actitudes y discute los métodos de las ciencias naturales para tales efectos, al hilo de la idea de "formación":

En efecto, la formación no consiste en que el que aprende acumule materias y métodos científicos, sino en que se forme a sí mismo [...] Y, así, la cultura, la formación, se entendía como el modo específicamente humano de dar forma a las disposiciones y capacidades naturales del hombre [...]. Conceder vigencia a las ideas de otra persona, en eso consiste la verdadera cultura y formación, porque presupone elevarse sobre la propia limitación (Grondin 2003).

De estas ideas quisiéramos extraer un par de orientaciones para el tema que nos ocupa. Formar implica el proceso continuo de estarse formando, por lo que nos encontramos con un círculo necesario que la educación debe considerar. Y en este preciso sentido la educación se pone al servicio de la generación de cultura, en la medida en que en ella se cultiva, partiendo por los profesores y directivos, una presencia abierta y respetuosa capaz de generar encuentros y diálogos colaborativos y creativos para un mundo complejo. Esta preciosa capacidad humana de abrirse a la posibilidad de que el otro (y lo otro) tenga algo que enseñarme, es clave, pues supone una capacidad para observar con claridad y para actuar con la compasión que surge desde la consciencia de una humanidad compartida. Creemos que esto sugiere un giro paradigmático en nuestra educación actual, que tenga como uno de sus ejes claves la calidad de la presencia del profesorado, presencia que puede ser cultivada desde un enfoque mindfulness capaz de permear el sistema educativo en su conjunto, basado tanto en las evidencias científicas con que contamos, así como en una 
reflexión profunda y amplia acerca del sentido del proceso formativo y de la educación en su conjunto en el contexto actual. La educación de una persona es un ámbito del quehacer humano donde conviene mantener presente aquella simple y fecunda idea según la cual hay veces en que la mejor forma de hacer es ser.

\section{REFERENCIAS BIBLIOGRÁFICAS}

Araya, C. y Moncada, L. (2016). Auto-compasión: origen, concepto y evidencias preliminares. Revista Argentina de Clínica Psicológica, XXV (1), 67-78.

Arellano, J. (2001). La reforma educacional chilena. Revista de la CEPAL, 73, 83-94.

Arguís, R. (2014). Mindfulness y educación. Aprendiendo a vivir con atención plena. En Cebolla, García-Campayo y Demarzo (Ed.), Mindfulness y ciencia. De la tradición a la modernidad (pp. 60-71). Madrid: Alianza Editorial.

Arón, A. y Milicic. N. (1999). Desgaste profesional de los profesores y clima social escolar. Recuperado el 16 de enero de 2017 desde http://www.buentrato.cl/pdf/est_inv/desgas/dpa_ aron1.pdf

Ausubel, N. (1983). Psicología educativa: Un punto de vista cognoscitivo. México: Editorial Trillas.

Bachrach, E. (2014). Encambio. Aprendé a modificar tu cerebro para cambiar tu vida y sentirte mejor. Buenos Aires: Sudamericana.

Baer, R. A. (2003). Mindfulness training as a clinical intervention: A conceptual and empirical review. Clinical Psychology: Science and Practice, 10, 351-366.

Beshai, S., McAlpine, L., Weare, K. \& Kuyken, W. A. (2016). Non-Randomised Feasibility Trial Assessing the Efficacy of a Mindfulness-Based Intervention for Teachers to Reduce Stress and Improve Well-Being. Mindfulness, 7 (1), 98-208.

Brito, G., Segú, C. y Araya, C. (2014). Presencia plena. Reflexiones y prácticas para cultivar mindfulness en la vida cotidiana. Santiago: J. C. Sáez Editor.

Brito, R. (2011). Habitar poético y presencia-atención plena (Mindfulness): un diálogo entre Occidente y Oriente. Gaceta de Psiquiatría Universitaria, 7 (3), 309-317.

Brito, R. (2015a). De la actitud a la cosmovisión terapéutica: propuesta de un modelo psico-sófico. Primera Parte. Gaceta de Psiquiatría Universitaria, 11 (3), 275-283.

Brito, R. (2015b). De la actitud a la cosmovisión terapéutica: Propuesta de un modelo psico-sófico. Segunda Parte. Gaceta de Psiquiatría Universitaria, 11 (4), 353-367.

Brown, K.W., Ryan, R.M. \& Creswell, J.D. (2007). Mindfulness: Theoretical foundations and evidence for its salutary effects. Psychological Inquiry, 18, 1-26.

Canales, I. y Rovira, G. (2016). La importancia de la autorregulación y la empatía en la formación docente. Red de Revistas Científicas de América Latina y el Caribe, España y Portugal, 22 (1), $157-172$.

Casassus, J. (2002). Cambios paradigmáticos en educación. Revista Brasileira de Educação, 20, 48-59.

Cebolla, García-Campayo y Demarzo (2014). Mindfulness y ciencia. De la tradición a la modernidad. Madrid: Alianza Editorial.

Cornejo, C. R. (2009). Condiciones de trabajo y bienestar/malestar docente en profesores de enseñanza media de Santiago de Chile. Educação e Sociedade, 30 (107), 409-426.

Cullen, M. y Brito, G. (2016). Mindfulness y equilibrio emocional. Un programa de ocho semanas para mejorar la salud emocional y aumentar la resiliencia. Málaga: Editorial SIRIO.

Delgado, L., Guerra, P., Perakakis, P., Viedma, M.I., Robles, H. y Vila, J. (2010). Eficacia de un programa de entrenamiento en conciencia plena (mindfulness) y valores humanos como herramienta de regulacion emocional y prevencion del estres para profesores. Behavioural Psychology/Psicologia Conductual, 18 (3), 511-532. 
Doherty, G., Forer, B., Lero, D., Goelman, H. y LaGrange, A. (2006). Predictors of quality in family child care. Early Childhood Research Quarterly, 21 (3), 296-312.

Flook, L; Goldberg, S; Pinger, L; Bonus, K \& Davidson, R. (2013). Mindfulness for teachers: A pilot study to assess effects on stress, burnout and teaching efficacy. Mind, brain and education, 7 (3). DOI:10.1111/mbe.12026. Recuperado el 15 de junio de 2017 desde https://www.ncbi.nlm.nih. gov/pmc/articles/PMC3855679/

Franco, C., Manas, I., Cangas, A. J., Moreno, E. y Gallego, J. (2010). Reducing teachers psychological distress through mindfulness training. Spanish Journal of Psychology, 13 (2), 655-666.

Gaete, A. ; Castro, M. ; Pino, F. y Mansilla, D. (2017). Abandono de la profesión docente en Chile : Motivos para irse del aula y condiciones para volver. Estudios pedagógicos, XLIII (1), pp. 123138.

Gadamer, H. G. (1994). Verdad y método II. Fundamentos de una hermenéutica filosófica. Salamanca: Sígueme.

Germer, C. \& Siegel, D. (2012). Wisdom and compassion in psychotherapy. Deeping mindfulness in clinical Practice. New York: Guilford Press.

Greeson, J. M. (2009). Mindfulness Research Update: 2008. Complementary Health Practice Review, 14 (1), 10-18.

Grondin, J. (2003). Introducción a Gadamer. Barcelona: Herder Editorial.

Grossman, P., Niemann, L., Schmidt, S. \& Walach, H. (2004). Mindfulness-based stress reduction and health benefits: A meta-analysis. Journal of Psychosomatic Research, 57, 35-43.

Han, B. (2012). La sociedad del cansancio. Barcelona: Herder Editorial.

Hoffman, S., Sawyer, A., Witt, A., \& Oh, D. (2010). The effect of mindfulness-based therapy on anxiety and depression: A meta-analytic review. Journal of Consulting and Clinical Psychology, 78 (2), 169-183.

Jennings, P. \& Greenber, M. (2013). Improving Classroom Learning Environments by Cultivating Awareness and Resilience in Education (CARE): Results of a Randomized Controlled Trial. School Psychology Quarterly, 28 (4), 374-390.

Kabat-Zinn, J. (2005). Coming to our senses: healing ourselves and the world through Mindfulness. New York: Hyperion.

Kabat-Zinn, J. (2013). Mindfulness para principiantes. Barcelona: Kairós.

Kabat-Zinn, J. (2016). Vivir con plenitud las crisis: cómo utilizar la sabiduría del cuerpo y de la mente para afrontar el estrés, el dolor y la enfermedad ( $2^{\circ}$ ed.). Barcelona: Kairós.

Kemeny, M. E., Foltz, C., Cavanagh, J. F., Cullen, M., Giese-Davis, J., Jennings, P., Rosenberg, E. L., Gillath, O., Shaver, P. R., Wallace, B. A., \& Ekman, P. (2011). Contemplative/emotion training reduces negative emotional behaviour and promotes prosocial responses. Emotion, 12 (2), 338-350.

Mañas, I., Franco, C. y Justo, E. (2011). Reducción de los niveles de estrés docente y los días de baja laboral por enfermedad en profesores de educación secundaria obligatoria a través de un programa de entrenamiento en mindfulness. Clínica y Salud, 22 (2), 121-137.

Mañas, I., Franco, C., Gil, M. y Gil, C. (2014). Educación consciente: Mindfulness (atención plena) en el ámbito educativo. Educadores conscientes formando a seres humanos conscientes. En Soriano, R. y Cruz, P. (Eds.). Alianza de civilizaciones, políticas migratorias y educación. Sevilla: Aconcagua Libros. Recuperado el 21 de noviembre de 2017 desde https://es.scribd. com/document/259982181/Atencion-Plena-en-La-Escuela

Meiklejohn, J., Phillips, C., Lee, M., Lee, M., Biegel, G., Roach, A., Frank, J., Burke, C., Pinger, L., Soloway, G., Isberg, R., Sibinga, E., Grossman, L. \& Saltzman, A. (2012). Integrating mindfulness training into K-12 education: Fostering the resilience of teachers and students. Mindfulness, 1 (1), pp. 291-307. DOI 10.1007/s12671-012-0094-5. Recuperado el 26 de enero de 2017 desde https://greatergood.berkeley.edu/images/uploads/Integrating_Mindfulness_ Training_Into_K-12_Education.pdf 
Mindful Nation UK (2015). Report by the Mindfulness All-Party Parliamentary Group (MAPPG). Recuperado el 3 de enero de 2017 desde www.themindfulnessinitiative.org.uk

Naranjo, C. (2007). Cambiar la educación para cambiar el mundo. Santiago: Cuarto Propio.

Neff, K. (2012). The Science of self-compassion. In Siegel, C. (Ed.), Compassion and Wisdom in Psychotherapy. New York: Guilford Press (pp. 79-92).

Ricard, M. (2016). En defensa del altruismo. Barcelona: Urano.

Robinson, K. (2009). The element. How finding your passion changes everything. New York: Viking.

Roeser, R., Skinner, E., Beers, J. \& Jennings, P. (2012). Mindfulness Training and Teachers Professional Development: An Emerging Area of Research and Practice. Child Development Perspectives, 6 (2), 167-173.

Rogers, C. (1989). El proceso de convertirse en persona. Barcelona: Editorial Paidós.

Shapiro, S; Brown, K. \& Astin, J. (2011). Toward the integration of meditation into higher education: A review of research, Teachers College Record, 113 (3), 493-528.

Siegel, D. (2012). Mindfulness y psicoterapia. Buenos Aires: Paidós.

Stahl, B. y Goldstein, E. (2010). Mindfulness para reducir el estrés. Una guía práctica. Barcelona: Kairós.

Tse, Lao (1990). Tao Te King. El libro del Tao y su Virtud. Santiago: Editorial Cuatro Vientos.

Valdivia, G., Avendaño, C., Bastías, S., Milicic N., Morales, A., Scharager., J. (2003). Estudio de salud laboral de los docentes en Chile. Escuela de Medicina y Psicología. Facultad de Medicina y Ciencias Sociales. Santiago: Pontificia Universidad Católica de Chile. Recuperado el 23 de enero de 2017 desde http://www.opech.cl/bibliografico/Doc_Docente/Estudio\%20de\%20 Salud\%20Laboral\%20de\%20Profesores\%20en\%20Chile.pd

Varela, F. (1998). Ética y acción. Santiago: Dolmen

Weare, K. (2014). Evidence for mindfulness: Impacts for wellbeing and performance of school staff. Mindfulness in Schools Project, 1-23. Recuperado el 7 de septiembre de 2017 desde https:// mindfulnessinschools.org

Williams, M. y Penman, D. (2013). Mindfulness. Guía práctica para encontrar la paz en un mundo frenético. Barcelona: Paidós.

Zenner, C., Herrnleben-Kurz, S. \& Walach, H. (2014). Mindfulness-based interventions in schools-a systematic review and meta-analysis. Frontiers in Psychology, 5, 1-20. DOI: 10.3389/ fpsyg.2014.00603. Recuperado el 9 de septiembre de 2017 desde file:///R:/Downloads/ fpsyg-05-00603.pdf 